\title{
A Theory of Asynchronous Control with Low Information Capacity Interfaces
}

\author{
J. Silva ${ }^{1}$, M. Dzura ${ }^{1}$, A. Mihailidis ${ }^{2}$ and T. Chau ${ }^{1}$ \\ ${ }^{1}$ Paediatric Rehabilitation Intelligent Systems Multidisciplinary Lab, Bloorview Research Institute, Toronto, Canada \\ ${ }^{2}$ Intelligent Assistive Technology and Systems Lab, University of Toronto, Toronto, Canada
}

\begin{abstract}
Low information capacity interfaces (LICIs) such as binary switches, often become the only means by which people with disabilities can interact with their assistive devices. Although numerous studies have been done to develop novel ways to estimate user intention in cases of extreme disability, little attention has been paid to the process by which the acquired information is translated into specific control tasks (e.g. navigating a menu or positioning a cursor). It has been traditionally assumed that an exact estimate of user intention is needed to produce reliable control commands. When using LICIs, this requires lengthy synchronous control strategies which demand a significant amount of sensory feedback. In order to develop appropriate alternatives, we have redefined the latter as a communication, rather than a control problem. Thus, our objective becomes the efficient transmission of control commands from the user to the device through LICIs. By applying basic principles of information theory, we use the natural uncertainty of asynchronous human-machine interactions with LICIs to obtain increasingly accurate estimates of user intention. Our theory is based on the implementation of a virtual intention pointer (VIP) that maps the user's intention on the device domain. Thus, changes in the VIP's behavior result in corresponding changes to the device being controlled. The VIP's behavior is described by user intention rings (UIRs). UIRs are unidimensional, viscoelastic spaces, mapped on each of the angular dimensions of the VIP's velocity vector. A variety of kernels representing different communication/control strategies may be used to deform the UIRs creating nonparametric estimates of user intention. Although cursor positioning and trajectory following in 2- and 3-D spaces are ideal applications for this theory, it can also be used for virtual navigation in multidimensional domains to control, for instance, prosthetic or robotic arms with multiple (>3) degrees of freedom.
\end{abstract}

Keywords - Asynchronous control, single-switch, access, human-machine interface.

\section{INTRODUCTION}

Interfaces may be defined as the point of interaction or communication between two entities. Thus, they can be seen as "translators" that obtain information from one entity and present it to the other in an appropriate and understandable manner. This transmission of information in the form of messages sent through a communication channel (i.e. the interface) constitutes the classical communication system as proposed by Shannon (1948) [1]

The fundamental underpinning of Shannon's work, which founded the field of information theory, is the realization of the relationship between the limits of communication efficiency and the entropy of the system in which the information exchange takes place. This suggests that common messages should be coded with as few information-carrying units or "bits" as possible in order to reduce the mean information transmission rate and, therefore, the overall cost (e.g. time, energy and bandwidth) of the transmission. Therefore, historical or contextual knowledge about the nature of the communication is directly associated with a reduction in the system's entropy, thus, allowing optimization.

The theory we propose, relates to the study of a special case of human-machine communication (see Figure 1). Namely, the efficient delivery of user commands to assistive devices by means of low information capacity interfaces (LICIs). This is an extremely common and, for the most part, still unresolved issue in rehabilitation engineering. Moreover, no theory has yet been identified or defined, let alone, systematically applied to the development and assessment of optimal control strategies for assistive devices using LICIs.

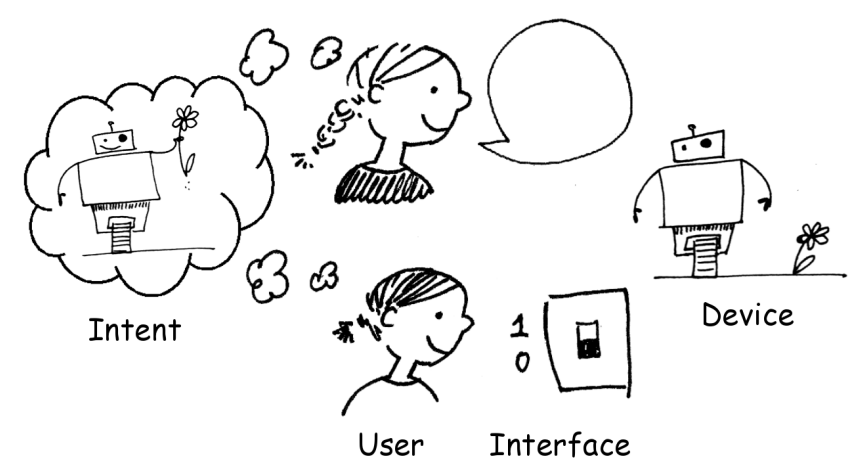

Fig. 1 The control paradigm as a communication system. The interface (i.e. the channel) may be used to communicate intent from the user to the device. The user on the top has access to a high information capacity interface (i.e. speech). The user on the bottom has access to a low information capacity interface (i.e. a binary switch). 
In the proposed paradigm, the challenge of using a low capacity channel (i.e. the interface) to "transmit" the intention of the user to the device is cast as a classical communication problem. A similar approach has recently been considered in the study of brain-computer interfaces (BCIs) [2, 3]. Here, the problem is analogous to that proposed by Shannon [1]. However, little to no attention has been given to the following step in the communication process: given one or more signals that can be reliably controlled by the user, what is the most efficient way to map these to actual device actions? Current non-invasive BCIs have an information capacity of about $0.5 \mathrm{bits} / \mathrm{s}$ [4]. This means the user would only be allowed to answer one "yes/no" question every two seconds. Thus, we begin to realize the need for efficient strategies that optimize the interaction between the user and the machine.

\section{Synchronous Communication}

Access strategies for people with disabilities are predominantly based on synchronous interactions with their devices. Typically, the user must wait for an external cue in order to communicate intention [5]. In the case of a single binary switch interface and a domain of three tasks, a typical interaction would involve the following steps:

1. Machine asks: "Do you want task A?" then waits for a switch press for a pre-defined period.

2. Machine asks: "Do you want task B?" then waits for a switch press for a pre-defined period.

3. Machine asks: "Do you want task C?" then waits for a switch press for a pre-defined period.

The process is then repeated. If the user activates the switch, the device assumes that the user's intention is to perform the task the machine asked about most recently. This recursive form of interaction is typically termed "single-switch scanning", and represents a means of indirect access to the desired command. However the user often has the cognitive capacity to state her/his intention directly without the need for sequential prompts. Unfortunately, the physical means to actualize this direct access is not usually available. Thus, while indirect access is facilitated through synchronous interactions, it comes with a high cost to the user, who must deal with lengthy wait times and constrained interactions since, typically, not all of the features of a given device can be made available to the user.

From an information theoretic perspective, synchronous communication strategies are also highly inefficient since they are based on assumptions that are not only inadequately substantiated, but are likely to be false. For instance:
1. Synchronous communication assumes that the a priori probability of choice over the task domain always follows a particular order. In our previous example, the machine will never ask about task $\mathrm{C}$ before asking about task B. Thus, in cases where the a priori probability of task B is actually higher than that of task C, the opportunity for optimization will be lost.

2. Synchronous communication assumes that the tasks in the domain are uncorrelated and independent random variables. This assumption makes previous choices independent of current and future choices. However, it is evident that historical information may be used to determine the likelihood of future choices.

\section{AsynCronous CONTROL WITH LICIS}

\section{A. Basic concepts}

Our theory for asynchronous control is based on the optimization of the process by which intention is communicated from a given user to a device being controlled. This requires the minimization of the number of information units (i.e. bits) required from the user to control the device. When using LICIs, this may be naturally achieved by implementing a control strategy that requires users to activate their interfaces only when their devices behave erroneously. In other words, rather than requiring an exact value of user intention, through a process of negative reinforcement, such strategy would use consecutive interface activations to obtain increasingly accurate estimates of user intention. This approach would not only optimize control by minimizing the number of interface activations required to control a device but would also provide unconstrained access to its full domain of activity. Thus, for instance, in the task of positioning a pointer on a multidimensional space, the user would not only have control over the final position of the pointer, but also over the particular trajectory followed by the latter.

The diagram in figure 2 presents the main components of our theory for asynchronous control with LICIs. In order to best illustrate it, we use single-switch navigation and pointer positioning on a 2-D domain as a sample application. Our theory is based on the implementation of a virtual intention pointer (VIP) that maps the user's intention on the device domain. Thus, changes in the VIP's behavior result in corresponding changes to the device being controlled. In our 2-D navigation case, the real pointer and the VIP are the same. The VIP's behavior is governed by user intention rings (UIRs). UIRs are unidimensional spaces, mapped on each of the angular dimensions of the spherical coordinates of the VIP's velocity vector $\vec{V}$. As depicted in figure 2 , in 




Fig. 2 A theory of asynchronous control with LICIs. A virtual intention pointer (VIP) mapped on a device domain will allow for unconstrained control of the device. With each binary activation, the LICI will deform the user intention ring (UIR) according to a particular radial kernel $g(\theta)$, updating the non-parametric estimate $f(\theta)$ of user intention.

the case of navigation on a 2-D domain, there is only one UIR corresponding to one angular dimension $\theta$ of $\vec{V}$. The magnitude of the VIP's velocity vector is set to a constant $c$. The gray color scale on the UIR represents varying degrees of belief $f(\theta)$ about the user's intention to avoid a given angle $\theta$. Higher values (i.e. darker colors) represent stronger assumptions. Thus, the value of $\theta$ that minimizes $f(\theta)$ represents the best guess for the intended angle $\varphi$ of the pointer velocity vector $\vec{V}$.

$$
\begin{gathered}
\varphi=\underset{\theta}{\arg \min } f(\theta) \\
\vec{V}=(c, \varphi)
\end{gathered}
$$

When the user activates the LICI to produce a single binary command, a radial intention kernel $g(\theta)$ is used to deform the UIR and, therefore, modify the belief $f(\theta)$. A variety of radial kernels, representing different communication/control strategies, may be used. Figure 2 shows a cosine kernel of the form:

$$
g(\theta)=\frac{1}{2}(\cos (\theta-\varphi)+1)
$$

This kernel represents the assumption that the current angle $\varphi$ will be the least preferred direction (i.e., the strongest belief of being erroneous) in the following activation of the LICI, while the opposite angle (i.e. $\varphi \pm 180$ ) will be given preference. However, the actual value of the angle chosen will depend on the particular state of the UIR according to equation 1 .
The radial kernel $g(\theta)$ deforms the UIR according to the following relationship:

$$
f(\theta)_{t}=f(\theta)_{t-\Delta t}+g(\theta)\left(1-f(\theta)_{t-\Delta t}\right)
$$

where $f(\theta)_{t}$ is the current estimate of $f(\theta)$ and $f(\theta)_{t-\Delta t}$ is the previous estimate. Since the previous belief estimate, $f(\theta)$. $\Delta t \in[0,1]$, a radial kernel, $g(\theta)$, with values between 0 and 1 , ensures that this deformation of the UIR results in a new belief estimate in the same range, i.e., $0 \leq f(\theta)_{t} \leq 1$.

Finally, in order to reduce the influence of the oldest events on future choices of $\varphi$ the UIR must be allowed to "forget" past deformations. This memory effect may be achieved by assigning some level of viscoelasticity to the UIR. The following recursive filter may be used for that purpose:

$$
f(\theta)_{t}=k \cdot f(\theta)_{t-\Delta t}
$$

where $k \in[0,1]$ is a constant directly proportional to the size of the memory window.

\section{B. Implementation}

We have focused our discussion on the description of this theory as a recursive process for the generation of on-line, non-parametric estimates of user intention. In order to further facilitate the application of the concepts presented, we will summarize the required steps for their implementation. Adhering to the example of 2-D navigation, a sample algorithm would involve the following sequential steps:

1. Originally, nothing is known about the user's intention regarding the VIP's behavior. Thus, on the first binary LICI activation, the angle $\varphi$ must be selected randomly from a uniform distribution.

2. This initial random choice of $\varphi$ will also determine the initial state $f(\theta)_{t=0}$ of the UIR. Thus,

$$
f(\theta)_{t=0}=g(\theta)
$$

3. From here, the filter described in equation 5 may be applied recursively. This filter causes an exponential decay on $f(\theta)$ (i.e. the "viscoelastic" effect), which effectively reduces the influence of the oldest events on future choices of $\varphi$.

4. For each of the future binary activations of the LICI, the UIR will be instantly deformed according to equation 4 . The estimate of user intention $\varphi$ will thus be updated according to equation 1 and the behavior of the VIP will be modified according to equation 2 . 


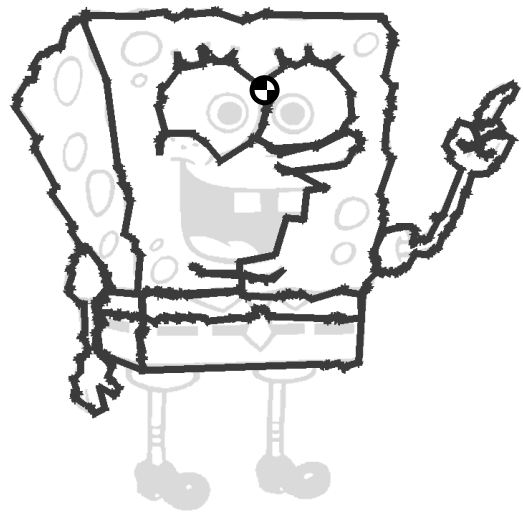

Fig. 3 Sample frame obtained from a single-switch, trajectory following (drawing) task using the algorithm presented. The black bold line represents the user-controlled pointer trajectory. The drawing in gray represents the "target" trajectory. The checkered circle indicates the current pointer position.

\section{Applications}

\section{A. Trajectory following}

There has been some debate in the last few years regarding the need for mouse emulation in computer systems for people with disabilities. In 1997, Shein [6] introduced the concept of task transparency and used it to suggest that mouse emulation is, for the most part, not necessary for people with disabilities since pointing is usually associated to a different underlying goal (e.g. selecting an item within a menu). In other words, pointing is not usually a goal in itself. However, Shein includes drawing as one of the few exceptions since; in this case, it is not only necessary to position a pointer in space, but also to follow a specific trajectory. Figure 3 shows a sample frame obtained from a single-switch drawing task implemented through the application of the theory presented in the previous section. The bold black line represents the user-controlled pointer trajectory. The drawing in gray represents the intended "target". To our knowledge, this is the first time that unconstrained, asynchronous single-switch drawing is enabled.

\section{B. Other applications}

Evidently, cursor positioning and trajectory following are ideal applications for our theory. The expansion of the algorithms presented here to navigation in a 3-D space, when combined with the use of inverse kinematics, may allow, for example, unconstrained control of the end point of a prosthetic or robotic arm with multiple (>3) degrees of freedom. But most importantly, our theory may be applied to any control paradigm where the domain of activity can be arranged in a navigable multidimensional domain. For example, the 2-D navigation algorithm presented here may be used in combination with the open source program Dasher [7] for the generation of text using a single binary switch. Furthermore, the appropriate arrangement of a multidimensional frequency space may allow, for instance, real-time music composition using LICIs.

\section{Conclusions}

A theory of asynchronous control with LICIs has been presented. The former is based on the optimized navigation of real and virtual multidimensional domains using LICIs as communication channels. For the first time, such theoretical framework supports the development of control strategies that allow unconstrained access to complex devices by means of LICIs. We believe this theory will revolutionize the concept of single-switch access and provide a wide range of possibilities for other access methods such as BCIs. As it has been demonstrated, the initial application of our theory for tracking pre-defined trajectories has resulted in the development of the first-ever, asynchronous singleswitch drawing application. However, additional work is required for the systematic characterization of the potential benefits and limitations of our theory. For instance, the development of a method for the design of optimal radial kernels for specific applications would be particularly useful.

\section{REFERENCES}

1. Shannon C E. (1948) A mathematical theory of communication. The Bell Sys Tech J 27:379-423

2. Millan J R, Renkens F et al (2004) Brain-actuated interaction. Artificial Intelligence 159:241-259

3. Taylor D M, Hells Tillery S I, Schwartz A B (2003) Information conveyed through brain control: cursor versus robot. IEEE Trans Neur Sys Rehab Eng 11(2):195-199 DOI 10.10007/s002149800025

4. Wolpaw J R, Birbaumer N et al (2002) Brain computer interfaces for communication and control. Clinical Neurophysiology 113:767-791

5. Cook A M, Hussey S M. (2002) Assistive technologies: principles and practice. Mosby Inc.

6. Shein G F (1997) Towards task transparency in alternative computer access: Selection of text through single-switch scanning. PhD dissertation, University of Toronto.

7. Dasher at http://www.inference.phy.cam.ac.uk/dasher/

Address of the corresponding author:

Author: Jorge Silva

Institute: Bloorview Research Institute

Street: 150 Kilgour Rd.

City: Toronto, ON

Country: Canada

Email: jorge.silva@utoronto.ca 